\title{
Directional Light Emission from Propagating Surface Plasmons of Silver Nanowires
}

\author{
Zhipeng Li, ${ }^{\dagger}$ Feng Hao, ${ }^{\ddagger}$ Yingzhou Huang, ${ }^{\dagger}$ Yurui Fang, ${ }^{\dagger}$ Peter Nordlander, ${ }^{*, \neq}$ \\ and Hongxing $\mathrm{Xu}^{\star,+, \S}$
}

Beijing National Laboratory for Condensed Matter Physics, and Institute of Physics, Chinese Academy of Sciences, Box 603-146, 100190, Beijing, China, Department of Physics and Astronomy, Department of Electrical and Computer Engineering, Laboratory for Nanophotonics, Rice University, Houston, Texas 77005, Division of Solid State Physics/The Nanometer Consortium, Lund University, Box 118, S-22100, Lund, Sweden

Received August 14, 2009; Revised Manuscript Received August 25, 2009

\begin{abstract}
Thin metallic nanowires are highly promising candidates for plasmonic waveguides in photonic and electronic devices. We have observed that light from the end of a silver nanowire, following excitation of plasmons at the other end of the wire, is emitted in a cone of angles peaking at nominally $45-60^{\circ}$ from the nanowire axis, with virtually no light emitted along the direction of the nanowire. This surprising characteristic can be explained in a simple picture invoking Fabry-Pérot resonances of the forward- and back-propagating plasmons on the nanowire. This strongly angular-dependent emission is a critical property that must be considered when designing coupled nanowire-based photonic devices and systems.
\end{abstract}

Plasmonic waveguides have significant potential to be used as a key component in miniaturized optical devices at the nanometer scale, and in the integration of photonic circuits with electronics to overcome the limitations of bandwidth and data transmission rates of classical electrical interconnects. ${ }^{1-8}$ Such an integration of photonics and electronics and the miniaturization of optical devices at the nanometer size are of considerable current interest in nanophotonics. ${ }^{8}$ When surface plasmon polaritons (SPPs), ${ }^{9}$ the collective motion of free electrons, are excited in the plasmonic waveguides, they can be propagated at distances exceeding tens of micrometers, in nanometer-width geometries such as nanoparticle chains, ${ }^{10-12}$ metal stripes, ${ }^{13}$ grooves,${ }^{14}$ metal-insulator-metal structures, ${ }^{7,15}$ and nanowires. ${ }^{16-20}$ While intensive experimental and theoretical efforts have focused on improving the in-coupling efficiency of light ${ }^{3,19}$ and on how to reduce the propagation loss, ${ }^{4,21}$ relatively little is known about their light-emitting properties. ${ }^{22}$ This is critically important information for the design and development of SPP waveguides in integrated photonic or electronic devices and systems.

\footnotetext{
* Corresponding authors, nordland@ rice.edu and hxxu@ aphy.iphy.ac.cn.

${ }^{\dagger}$ Chinese Academy of Sciences.

* Rice University.

$\S$ Lund University.
}

In this paper, we measure the spatial distribution of the light emitted from one end of a nanowire following the excitation of SPPs at the other end. Surprisingly, we find that almost no light is emitted in the direction of the nanowire but instead peaked at nominally $45-60^{\circ}$ from the direction of the wire. For thin nanowires we observe that the distribution of the emitted light is remarkably insensitive to the diameter and length of the nanowire and the detailed structure of the wire ends.

Several different approaches have been developed for the imaging of plasmonic properties of metallic nanostructures. ${ }^{23,24}$ In the present work, the SPPs are excited by focusing a laser beam through an objective to one end of the nanowire, which is shown in Figure 1a. The emission from the other end of the nanowire is collected by the same objective and the optical image is recorded by a TE cooled $1392 \times 1040$ CCD detector mounted on a microscope (Olympus BX51). The intensity of the emission is determined by finding the maximum value in the emission spot from the optical image. The objective itself has an inside iris diaphragm which varies the numerical aperture (N.A.) from 0.5 to 1.2. As shown in Figure 1b, only the emission within the collection cone of the objective can be harvested and recorded by the CCD. Hence, the measurement of the emission intensity under different N.A. will contain informa- 


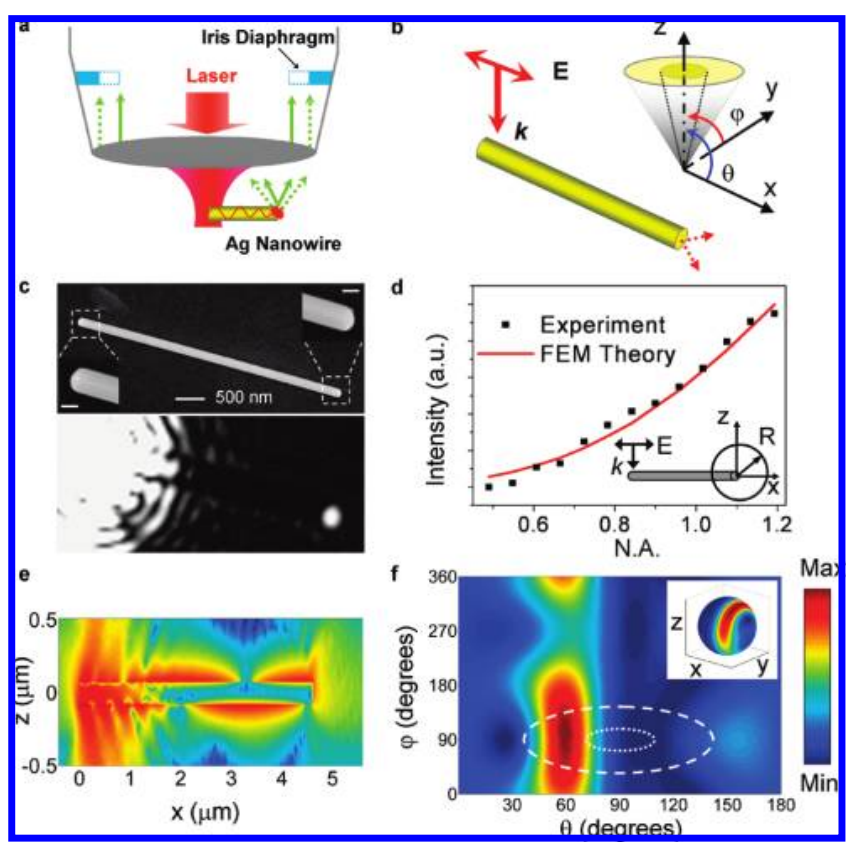

Figure 1. Angular emission measurement. (a) Scheme of the measurement. The excitation and collection by an objective (Olympus UPlanApo, $100 \times$, oil immersion $n=1.518$ ). The iris diaphragm inside the objective varies the N.A. from 1.2 to 0.5 during the measurements. (b) The coordinates referred to in the experiments. The wave vector and polarization of the incident light are represented by the red arrows. The different N.A. corresponds to different opening angles of the collection cone. The light that is emitted into the cone will be collected by the objective and recorded by the CCD. (c) The SEM image of a wire of length $4.6 \mu \mathrm{m}$ and diameter $158 \mathrm{~nm}$, and its optical image in a microscope under the excitation of a $633 \mathrm{~nm}$ laser spot polarized along the wire. The scale bar in the SEM insets showing the wire end are $100 \mathrm{~nm}$. (d) The measured emission intensity as a function of the N.A. of the objective (black squares). Red curve is the integrated far-field intensity for different N.A. obtained from the simulation. (e) The FEM calculated distribution of the Poynting intensity around the wire. (f) The calculated emission intensity as a function of angles $\phi$ and $\theta$ obtained by far-field transformation. The inset shows the corresponding angular distribution on the integration sphere. The white rings of different sizes are the emission angles that can be collected by the objective for N.A. $=1.2$ and 0.5 , respectively.

tion of the angular distribution of the emitted light. The details on the sample preparation and how the instrument was calibrated are presented in the Supporting Information.

Figure 1c shows a scanning electron microscopy (SEM) image of an $\mathrm{Ag}$ nanowire of length $4.6 \mu \mathrm{m}$ and diameter $158 \mathrm{~nm}$. The nanowire is synthesized by chemical fabrication, ${ }^{25}$ yielding crystalline nanowires with smooth surfaces which avoid the scattering of SPPs at surface defects. The wire is deposited on an ITO glass substrate and immersed in index matched oil to eliminate substrate reflections. One end of the wire is excited by a diffraction-limited spot of $633 \mathrm{~nm}$ laser to launch the propagating SPPs. The polarization of the laser is rotated parallel to the wire using a halfwave plate to ensure optimal coupling between the incident light and the nanowire. No polarizer is used in the detection of the emitted light. A bright spot of the emission can be clearly observed at the other end of the wire. The brightness of the emission is decreased gradually as the N.A. varies from 1.2 to 0.5, which is shown in Figure 1d. What should be noted is that the decrease of the emission intensity is not caused by the reduction of the power of the incident laser, since the beam size is smaller than the opening width of the diaphragm even when the smallest N.A. $=0.5$ is used. This is also confirmed by the direct measurement of the power of the focused light under the objective which shows no change observed when the N.A. varied from 1.2 to 0.5 . Hence, the experiment data in Figure 1d reflect the directional emission of light from the emission end of the nanowire. To understand the spatial distribution of the light emission, we perform electromagnetic simulations using the finite element method (FEM) based commercial COMSOL multiphysics software package. For simplicity, we treat the wire as a cylindrical structure of a length $4.6 \mu \mathrm{m}$ and a diameter $158 \mathrm{~nm}$. Figure 1e shows the intensity distribution of Poynting vectors around the wire. It can be seen that the SPPs are scattered to the far field at the end of the wire. The wavelength of the surface plasmons excited in the wire is about $\lambda_{\mathrm{s}}=360 \mathrm{~nm}$. To compare the calculation with the experiment, we calculate the far-field emission intensity on the surface of a sphere enclosing the emitting end of the wire. The radius of the sphere is $R=300 \mathrm{~nm}$ corresponding roughly to the size of the emission spot measured in the experiment. By integrating the emission intensity that falls into the collection cone corresponding to the different N.A., we reproduce the experiment with the curves shown in Figure 1d. The spatial distribution of the light emission on the $\mathrm{R}=$ $300 \mathrm{~nm}$ sphere is shown in Figure 1f. The white ellipses indicate the emission angles that can be collected within the collection cones corresponding to N.A.$=1.2$ and 0.5 . The light emitted from the nanowire waveguide peaks at an emission angle around $\theta \sim 60^{\circ}$. Due to retardation, the emission intensity is slightly inhomogeneous in the $\phi$ direction. In the Supporting Information, we show that the angular distribution patterns for several other thin nanowires of different geometries and tip termination geometries remain very similar to the wire studied in Figure 1 with emission peaks around $45-60^{\circ}$. To mimic the dielectric environment of the nanowire, all calculations are performed for a nanowire embedded in a homogeneous dielectric medium of refractive index 1.5. For a more inhomogeneous substrate such as a $100 \mathrm{~nm}$ ITO film on a glass substrate or a nanowire on a substrate without the index matched oil, the calculated wire emission characteristics are essentially the same.

For wires with diameters smaller than about half of the incident wavelength $\lambda_{\text {ind }} / 2$ (in the experiment the surrounding medium is oil), the intensity of the emitted light is almost homogeneous in the $\phi$ direction. Hence, only the emission distribution in the $\theta$ direction needs to be considered. In Figure 2a, we show how the emission distribution depends on the length of the wire for a fixed diameter. The calculation shows that as the length of the wire increases, the predominant emission angle is always around $\sim 50^{\circ}$ with secondary peaks appearing at $\sim 140^{\circ}$, which is consistent with the experimental results in Figure 1 and Figure S3 in the Supporting Information. The maximum of the emission intensity exhibits periodic oscillations with wire length. The 


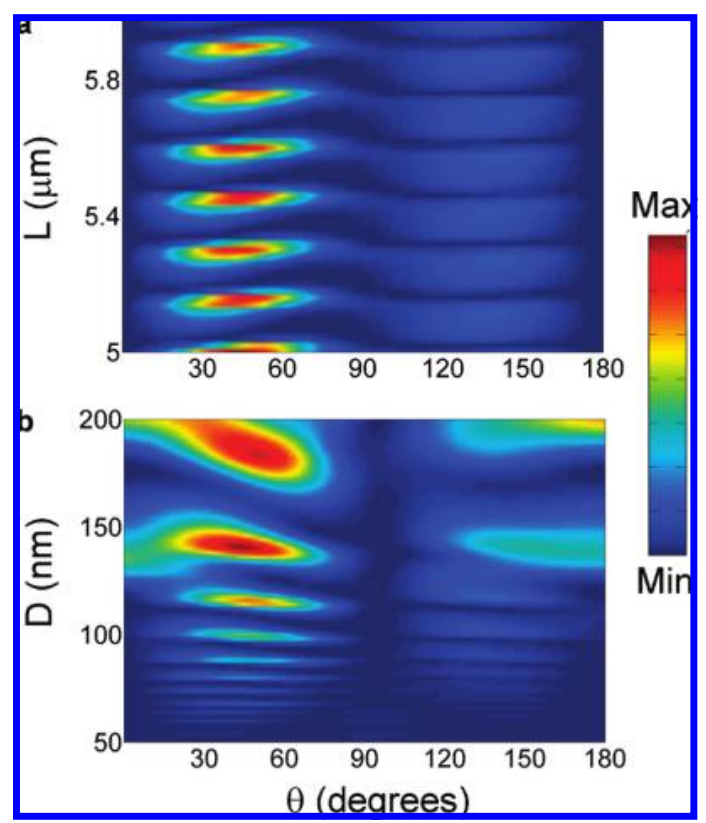

Figure 2. The dependence of the emission on the length and diameter of the wire. (a) The calculated emission intensity in the $\theta$ direction for a thin wire $(D=80 \mathrm{~nm})$ as a function of the wire length. (b) The calculated emission intensity in the $\theta$ direction for a wire of length $L=5 \mu \mathrm{m}$ as a function of the diameter of the wire. The wires are excited by a $633 \mathrm{~nm}$ laser spot which is polarized parallel to the wire. The emission intensity in the $\theta$ direction is obtained by integrating the emitted light on a $R=300$ $\mathrm{nm}$ sphere along the $\phi$ direction.

period is about $150 \mathrm{~nm}$, which is half of the wavelength of the propagating SPPs in the wire $\left(\lambda_{\mathrm{s}} \sim 300 \mathrm{~nm}\right.$ for the wire with $D=80 \mathrm{~nm}$ ). This observation suggests that the emission maximum from the wire occurs when standing waves of the surface plasmons are formed by the internal reflections from the wire ends. The maximum of the emission intensity thus corresponds to the formation of such Fabry-Pérot (FP) resonances. ${ }^{26,27}$

In Figure $2 \mathrm{~b}$ we show how the emission distribution depends on wire diameter for a wire of fixed length $L=5$ $\mu \mathrm{m}$ with the diameters ranging from 50 to $200 \mathrm{~nm}$, which is smaller than the half of the incident wavelength in oil (633 $\mathrm{nm}$ excitation). It is shown that the light emission is peaked at angles $\theta$ in the range of $45-60^{\circ}$ with a secondary emission peak at $130-150^{\circ}$. Similar to Figure 2a, the maximum of the emission intensity exhibits oscillations as the diameter $D$ is increased and are caused by the formation of FP resonances in the nanowires. ${ }^{26,27}$ The periodicity of these oscillations increases with the diameter $D$ because the wavelengths of wire plasmons of a fixed energy are proportional to $D .^{28}$

In the experiment we observed some variations of the emission intensities between different wires of similar diameters. These observations are consistent with the results in Figure 2 showing that the emission intensity depends sensitively on both the length and diameter of the wire.

As discussed above, the light emission originates from FP resonances in the nanowires and can be understood using a simple standing wave antenna current model $(\mathrm{CM})$. Here the

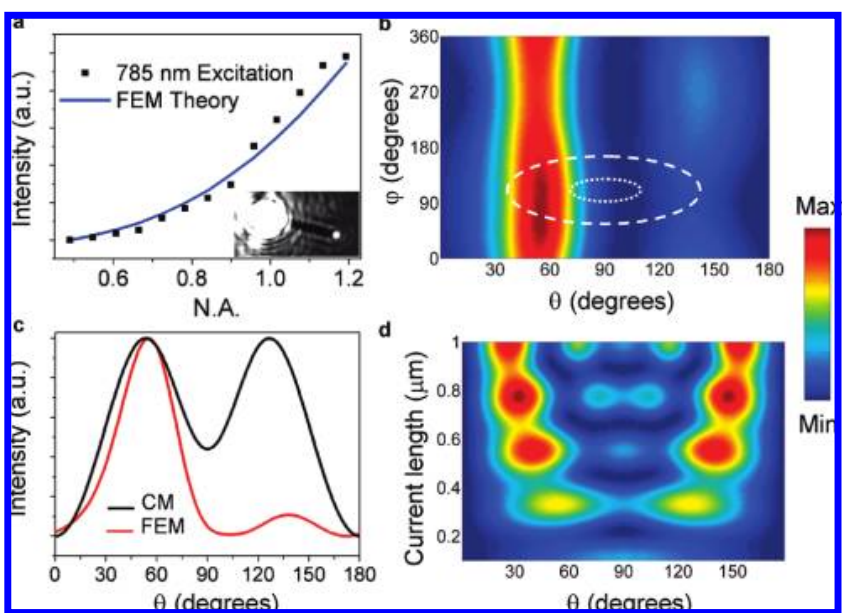

Figure 3. The current antenna model. (a) The measured emission intensity for the wire shown in Figure $1 \mathrm{c}$ with the same configuration as that in Figure 1d but with a different excitation of 785 $\mathrm{nm}$. Inset in (a) is the optical image obtained by the bright-field microscope. (b) The calculated emission intensity distribution on the integration sphere $(\mathrm{R}=400 \mathrm{~nm})$ obtained by far-field transformation. The white rings with different sizes are the range that can be collected by the objective for N.A. $=1.2$ and 0.5 , respectively. (c) The emission intensity along the $\theta$ direction for a $L=4.6 \mu \mathrm{m}, D=158 \mathrm{~nm}$ wire excited by a $785 \mathrm{~nm}$ laser spot polarized parallel to the wire. Red and black curves are calculated using FEM and the current model (CM), respectively. (d) The emission intensity along the $\theta$ direction from the standing wave current antenna with different current element lengths.

standing wave of surface plasmons is treated as the superpositions of two counterpropagating dipole currents. Parts a and $b$ of Figure 3 show the emission intensity as a function of different N.A. of the objective and the calculated distribution of the emission intensity in $\theta$ direction for the wire in Figure 1c excited by a $785 \mathrm{~nm}$ laser. The red and black curves in Figure $3 \mathrm{c}$ are the simulations obtained by the FEM method and the CM (Supporting Information), respectively. When the length of the current element is close to the radius of the integration sphere used in the FEM calculation $(\mathrm{R}=$ $400 \mathrm{~nm}$ for $785 \mathrm{~nm}$ excitation), the two simulations give similar emission distributions in the $\theta$ direction. In the $\mathrm{CM}$, there are two symmetric peaks with equal magnitude at around $60^{\circ}$ and $130^{\circ}$, whereas in the FEM calculation the two peaks are of unequal intensity. The discrepancy between the results from these two models originates from the energy loss induced by the propagation and the reflection of the surface plasmons at the end of the wire, which results in only a partial standing wave. In the CM, the magnitudes of the two superimposed traveling currents are assumed to be equal. Figure $3 d$ shows that the emission distribution in the $\theta$ direction is dependent on the current element length. The role of the current element length in the CM is the same as the radius of the integration sphere in FEM calculation. Hence, according to the condition of the experiment, a physical and reasonable value of the current element length will be about the size of the emission spot, which is confirmed by the consistency between the results from the experiment and the CM.

In conclusion, we have measured the angular dependence of the light emission from thin $\mathrm{Ag}$ nanowires: a prominent 
emission angle exists at nominally $60^{\circ}$ from the nanowire axis. The angular distribution of the light emission is found to be relatively independent of the shape and length of the wire and can be understood using a simple model invoking the Fabry-Pérot resonances of the nanowire waveguide. This light-emitting property will dictate the relative position and orientation of nanowire waveguides with respect to other optical and electronic elements in integrated optical and electronic devices.

Acknowledgment. This work was supported by NSFC GrantNo. 10625418, 10874233,MOSTGrantNo. 2006DFB02020, 2007CB936800, 2009CB930700, "Bairen Project" of CAS, and the Robert A. Welch foundation under Grant C-1222, the National Science Foundation under Grant CNS-0421109, and by the U.S. Army Research Office under Grant W911NF04-1-0203.

Supporting Information Available: Sample preparation, calibration of the N.A. of the objective, effect of the wire shape to the angular emission, more measurement for different wires, and the current antenna model. This material is available free of charge via the Internet at http:// pubs.acs.org.

\section{References}

(1) Lal, S.; Link, S.; Halas, N. J. Nat. Photonics 2007, 1, 641-648.

(2) Ozbay, E. Science 2006, 311, 189-193.

(3) Akimov, A. V.; Mukherjee, A.; Yu, C. L.; Chang, D. E.; Zibrov, A. S.; Hemmer, P. R.; Park, H.; Lukin, M. D. Nature 2007, 450, 402-406.

(4) Oulton, R. F.; Sorger, V. J.; Genov, D. A.; Pile, D. F. P.; Zhang, X. Nat. Photonics 2008, 2, 496-500.

(5) Bozhevolnyi, S. I.; Volkov, V. S.; Devaux, E.; Laluet, J. Y.; Ebbesen, T. W. Nature 2006, 440, 508-511.

(6) Ju, J. J.; Park, S.; Kim, M. S.; Kim, J. T.; Park, S. K.; Park, Y. J.; Lee, M. H. Appl. Phys. Lett. 2007, 91, 171117.
(7) Neutens, P.; Van Dorpe, P.; De Vlaminck, I.; Lagae, L.; Borghs, G. Nat. Photonics 2009, 3, 283-286.

(8) Zia, R.; Schuller, J. A.; Chandran, A.; Brongersma, M. L. Mater. Today 2006, 9, 20-27.

(9) Raether, H. H. Surface Plasmons; Springer: Berlin, 1988.

(10) Quinten, M.; Leitner, A.; Krenn, J. R.; Aussenegg, F. R. Opt. Lett. 1998, 23, 1331-1333.

(11) Maier, S. A.; Kik, P. G.; Atwater, H. A.; Meltzer, S.; Harel, E.; Koel, B. E.; Requicha, A. G. Nat. Mater. 2003, 2, 229-232.

(12) Li, Z. P.; Xu, H. X. J. Ouant. Spectrosc. Radiat. Transfer 2007, 103, 394-401.

(13) Lamprecht, B.; Krenn, J. R.; Schider, G.; Ditlbacher, H.; Salerno, M.; Felidj, N.; Leitner, A.; Aussenegg, F. R.; Weeber, J. C. Appl. Phvs. Lett. 2001, 79, 51-53.

(14) Volkov, V. S.; Bozhevolnyi, S. I.; Rodrigo, S. G.; Martin-Moreno, L.; Garcia-Vidal, F. J.; Devaux, E.; Ebbesen, T. W. Nano Lett. 2009, 9, 1278-1282.

(15) Verhagen, E.; Dionne, J. A.; Kuipers, L.; Atwater, H. A.; Polman, A. Nano Lett. 2008, 8, 2925-2929.

(16) Dickson, R. M.; Lyon, L. A. J.Phys. Chem. B 2000, 104, 60956098.

(17) Ditlbacher, H.; Hohenau, A.; Wagner, D.; Kreibig, U.; Rogers, M.; Hofer, F.; Aussenegg, F. R.; Krenn, J. R. Phvs. Rev. Lett. 2005, 95, 257403.

(18) Sanders, A. W.; Routenberg, D. A.; Wiley, B. J.; Xia, Y. N.; Dufresne, E. R.; Reed, M. A. Nano Lett. 2006, 6, 1822-1826.

(19) Knight, M. W.; Grady, N. K.; Bardhan, R.; Hao, F.; Nordlander, P.; Halas, N. J. Nano Lett. 2007, 7, 2346-2350.

(20) Fang, Y. R.; Wei, H.; Hao, F.; Nordlander, P.; Xu, H. X. Nano Lett. 2009, 9, 2049-2053.

(21) Manjavacas, A.; de Abajo, F. J. G. Nano Lett. 2009, 9, 1285-1289.

(22) Deibel, J. A.; Berndsen, N.; Wang, K. L.; Mittleman, D. M.; van der Valk, N. C. J.; Planken, P. C. M. Opt. Express 2006, 14, 8772-8778.

(23) Esteban, R.; Vogelgesang, R.; Dorfmuller, J.; Dmitriev, A.; Rockstuhl, C.; Etrich, C.; Kern, K. Nano Lett. 2008, 8, 3155-3159.

(24) Schaffer, B.; Hohenester, U.; Trugler, A.; Hofer, F. Phvs. Rev. B 2009, 79, 041401.

(25) Sun, Y. G.; Xia, Y. N. Adv. Mater. 2002, 14, 833-837.

(26) Vesseur, E. J. R.; de Waele, R.; Kuttge, M.; Polman, A. Nano Lett. 2007, 7, 2843-2846.

(27) Dorfmuller, J.; Vogelgesang, R.; Weitz, R. T.; Rockstuhl, C.; Etrich, C.; Pertsch, T.; Lederer, F.; Kern, K. Nano Lett. 2009, 9, 2372-2377.

(28) Hao, F.; Nordlander, P. Appl. Phvs. Lett. 2006, 89, 103101.

NL902651E 